\title{
Los maestros del trauma torácico en el Hospital Central de la Cruz Roja Mexicana. Su historia y legado
}

\author{
Juan Carlos Vázquez-Minero', Guillermo Vallejo-Sandoval', Anette Ochmann-Ratch ${ }^{3}$, \\ Laura Portillo-Téllez y Demian Trueba-Lozano ${ }^{4}$ \\ ${ }^{1}$ Cirujano general y cardiotorácico; ${ }^{2}$ Jefe del Servicio de Cirugía General y Urgencias; ${ }^{3}$ Director médico; ${ }^{4}$ Servicio de Cirugía General. Hospital \\ Central de la Cruz Roja Mexicana, Ciudad de México, México
}

\begin{abstract}
Resumen
La medicina encargada de la atención de víctimas de lesiones de diferentes tipos nació durante las guerras como consecuencia de la imperiosa necesidad de salvar vidas. En México, uno de los primeros hospitales dedicados a la atención de pacientes lesionados fue el Hospital Central de la Cruz Roja Mexicana. El objetivo de este artículo es hacer un homenaje al primer servicio para la atención de pacientes con traumatismo torácico, fundado en 1954, destacando a sus fundadores y a quienes han continuado con su labor: el Dr. Pedro Alegría Garza, el Dr. Octavio Rivas Solís, el Dr. Jesús Genis Becerra, etc. En 64 años de existencia se han formado ocho especialistas en cirugía cardiotorácica, de los cuales tres han laborado dentro del Hospital Central de la Cruz Roja Mexicana.
\end{abstract}

PALABRAS CLAVE: Cirugía cardiotorácica. Trauma. Cruz Roja.

\begin{abstract}
The branches of medicine responsible for the care of victims of different types of injuries were born as a result of the urgent need to save lives during the wars. In Mexico, one of the first hospitals developed for the care of injured patients was the Central Hospital of the Mexican Red Cross. The aim of this article is to pay tribute to the first service for the care of patients with chest trauma, founded in 1954, its founders and those who have continued with their work: Dr. Pedro Garza Alegría, Dr. Octavio Rivas Solís Dr. Jesús Genis Becerra, etc. In 64 years of existence, there have been trained eight specialists in cardiothoracic surgery and three of them have worked in the Central Hospital of the Mexican Red Cross.
\end{abstract}

KEY WORDS: Cardiothoracic surgery. Trauma. Red Cross.

\section{Antecedentes de hospitales especializados en la atención del trauma}

En 1847, al acercarse el ejército norteamericano a la Ciudad de México, el ayuntamiento local fundó dos centros hospitalarios para la atención de las víctimas del conflicto inminente. Estos hospitales se construyeron en la garita de San Lázaro y en el convento de San Pablo, y comenzaron a atender a los heridos de las batallas de Padierna y Churubusco a partir del 23 de agosto de ese año'.
Posteriormente, las deficiencias en la atención de los pacientes con lesiones derivadas de accidentes y agresiones en la ciudad llevaron a la necesidad de crear lugares especializados para su atención inmediata. Por este motivo, Leopoldo Castro creó el primer puesto de socorro en la calle de Revillagigedo, en 1908. Este espacio contaba con instrumental para llevar a cabo cirugía de urgencia y recursos para el traslado de pacientes. Este hecho marcó el inicio de la Cruz Verde ${ }^{1,2}$.

Correspondencia:

Juan Carlos Vázquez-Minero

Avenida Ejército Nacional, 1032

Col. Los Morales/Polanco

Fecha de recepción: 16-07-2016

C.P. 11510, Ciudad de México, México

Fecha de aceptación: 21-04-2017

E-mail: minerojc@hotmail.com

DOI://dx.doi.org/10.24875/GMM.17002766

Gac Med Mex. 2017;153:731-734

Contents available at PubMed

www.gacetamedicademexico.com 


\section{Antecedentes de la Cruz Roja Mexicana}

La historia de la Cruz Roja Mexicana se remonta hasta 1906, cuando el general Porfirio Díaz firmó el convenio en el cual México se unía a la convención de Ginebra de 1864, con el fin de mejorar la atención de los heridos y enfermos de los ejércitos en campaña. Dos años después, el 5 de junio de 1909, se instaló la primera mesa directiva de la Cruz Roja Mexicana.

La primera brigada de ayuda que enarboló el emblema de la Cruz Roja salió de la Ciudad de México en 1909 para asistir a las víctimas de la inundación que afectó Monterrey. Esta brigada fue encabezada por la Sra. Luz González Cosío de López y el Dr. Fernando López y Sánchez Román.

En 1910 se dio reconocimiento oficial a la Cruz Roja Mexicana -que en sus estatutos se denominaba Asociación Mexicana de la Cruz Roja-; este decreto se publicó en el Diario Oficial de la Federación el 12 de marzo de ese año, y el 26 de abril se fundó la primera mesa directiva. En 1911, el Comité Internacional de la Cruz Roja otorgó reconocimiento a la Cruz Roja Mexicana, y en 1923 fue reconocida por la Liga de Sociedades de la Cruz Roja y Media Luna Roja.

Los primeros servicios de ambulancias, así como la asistencia de primeros auxilios a las víctimas de las contiendas revolucionarias, se proporcionaron en la Ciudad de México ${ }^{3,4}$.

\section{Antecedentes de los hospitales de la Cruz Roja}

La creación de instalaciones para proporcionar atención a los pacientes ocurrió de la siguiente manera:

- 1909-1910: Artes No. 19 (Antonio Caso).

- 1910-1914: Rosales No. 20 (Torre Caballito).

- 1914-1916: Avenida Paseo de la Reforma No. 40 (junto a la Cámara de Comercio del Distrito Federal); San Francisco y Teatro Nacional (Eje Central y Madero), Edificio Guardiola (antiguo Edificio Escandón).

- 1916-1934: San Jerónimo No. 14 (Hospital Concepción Beistegui o de Regina o de San Jerónimo).

- 1934-1968: Durango y Monterrey (colonia Roma).

- 1968 hasta hoy: Avenida Ejército Nacional No. 1032, Colonia Los Morales/Polanco (Hospital Central).

Esta última ubicación del hospital está íntimamente ligada a las instalaciones de la Sede Nacional (Entidad Directiva Nacional de la Cruz Roja Mexicana, la cual estuvo alojada durante mucho tiempo dentro del mismo hospital). La Sede Nacional se ubica en la calle Juan Luis Vives No. 200, y la Delegación Distrito Federal está en Avenida Ejército Nacional No. 1032. En este lugar se llevaron a cabo las gestiones necesarias para la fundación de la entonces llamada Asociación Mexicana de la Cruz Roja, y se organizaron y coordinaron las primeras acciones de ayuda que proporcionó la institución ${ }^{3,4}$.

\section{Antecedentes de la cirugía torácica de trauma}

Es probable que la referencia más antigua del manejo del trauma torácico en América sea la narración que Alvar Núñez Cabeza de Vaca hizo de la extracción de una flecha del tórax de un indígena americano ${ }^{5}$. Existen muy pocos informes posteriores, hasta 1902, cuando el Dr. Adrián de Garay describió las heridas penetrantes de corazón. A continuación, en 1913, el Dr. Javier Ibarra reportó un intento para reparar una lesión cardiaca con malos resultados, y más adelante, en el Hospital Juárez, los doctores Rafael Vargas Otero, Clemente Robles Castillo, Fernando Valdés Villarreal y Manuel Godoy llevaron a cabo las primeras suturas cardiacas exitosas ${ }^{6}$.

Hasta 1870, la mortalidad del trauma torácico penetrante sin involucro de corazón o grandes vasos, con manejo expectante, era del 68\%; sin embargo, entre 1870 y 1898, cuando se efectuaron las primeras toracocentesis, pleurostomías y lavados, esta se redujo hasta el $20-30 \%$, y a partir de 1898, con la ligadura de vasos menores, disminuyó hasta el 7\%,8. Esta importante reducción en la mortalidad demostró que era necesario un manejo oportuno y especializado de los pacientes con este tipo de lesiones, lo cual devino en la necesidad de crear más hospitales con personal capacitado para esos fines.

\section{El servicio de traumatología torácica de la Cruz Roja Mexicana}

Desde su fundación, la Cruz Roja Mexicana ha proporcionado tratamiento a pacientes con trauma torácico. Esta atención fue brindada por cirujanos generales hasta la llegada del Dr. Pedro Alegría Garza, médico militar graduado en 1946, que trabajaba en el Sanatorio para Tuberculosos de Huipulco (hoy Instituto Nacional de Enfermedades Respiratorias) como neumólogo y cirujano de tórax.

El Dr. Alegría llegó a trabajar al viejo hospital de Monterrey y Durango, fundando en 1954, junto con los 
doctores Pablo Cruz Esparza (cirujano general y gastroenterólogo), Edmundo Ángeles y Gloria Eugenia Torres (neumóloga), también procedentes del Sanatorio para Tuberculosos de Huipulco, y fundó el primer servicio de cirugía torácica para casos de urgencia en un hospital, por invitación del Honorable Comité Médico de la Cruz Roja ante la necesidad de atención especializada de estos pacientes 9 .

El nuevo Hospital de la Cruz Roja Mexicana fue fundado el 16 de enero de 1968 a las 10:30 horas por el presidente de la República Lic. Gustavo Díaz Ordaz, nombrando como presidente de la institución a don José Barroso Chávez. En estas nuevas instalaciones continuó la labor del servicio de traumatología del tórax a cargo del Dr. Octavio Rivas Solís, pues el Dr. Pedro Alegría Torres fue designado coordinador de enseñanza ${ }^{10}$.

El Dr. Jesús Genis Becerra, originario de la Ciudad de México y graduado en 1956 de la Universidad Nacional Autónoma de México - aunque inicio sus estudios de medicina en la Escuela Médico Militar- cursó la especialidad en neumología y cirugía del tórax en el Instituto de Neumología y Cirugía del Tórax (actualmente Hospital General Manuel Gea González) de 1958 a 1961. En 1954, durante su etapa de pregrado, llevó a cabo sus prácticas de urgencias en el Hospital de la Cruz Roja, donde entró en contacto con la traumatología torácica como servicio especializado y nació su gusto por la cirugía torácica. Después de haber trabajado en el Instituto Mexicano del Seguro Social como neumólogo en 1971, volvió al Hospital de la Cruz Roja, fue contratado como especialista en cirugía torácica y trabajó durante un año con el Dr. Pedro Alegría, compartiendo las responsabilidades de la atención de los pacientes, efectuando la colocación y cuidados de sondas de pleurostomía, empleando sellos de agua de dos frascos y practicando las toracotomías en los pacientes que lo ameritaban en agudo o para la resolución de complicaciones ${ }^{11}$.

El Dr. Alegría trabajó durante un año más y se retiró en 1972 con 18 años de servicio en la Cruz Roja. El Dr. Octavio Rivas Solís, cirujano cardiovascular egresado del Instituto Nacional de Cardiología Ignacio Chávez, laboró en la Cruz Roja Mexicana desde su antigua sede en las calles de Monterrey y Durango, y continuó durante algunos años en el nuevo hospital de Polanco, atendiendo a los pacientes con trauma torácico y cardiovascular hasta 1972, cuando decidió dedicarse de lleno a la psicología deportiva, siendo un referente en esta materia hasta la fecha. A partir de ese año, el Dr. Genis quedó como encargado del servicio de traumatología torácica hasta 2008, cuando se retiró después de 36 años de servicio en la institución.

Estos tres pilares de la cirugía torácica mexicana no solo entregaron su valioso tiempo y dedicación para la fundación de un servicio y la atención de los pacientes, sino que también abrieron un semillero para nuevas generaciones.

\section{Nuevas generaciones}

Los hospitales cumplen un papel fundamental para las sociedades, pues son centros de atención y resolución de problemas, lugares de investigación y sedes para la formación de nuevos especialistas. En el Hospital Central de la Cruz Roja Mexicana se forman cirujanos generales, cuyo entrenamiento era de 3 años hasta el año 2000, a partir del cual la Universidad Nacional Autónoma de México lo incrementó a 4 años. Al concluir su entrenamiento, los médicos - muchos de ellos de provincia y algunos otros extranjeros-salen al campo laboral; sin embargo, algunos buscan mayor entrenamiento. El estrecho contacto con la patología traumática torácica contribuyó con el inicio del entrenamiento del primer especialista en cirugía cardiotorácica en 1990, el cual había recibido formación como cirujano general en la Cruz Roja Mexicana.

Como consecuencia del interés de los grandes cirujanos dedicados al trauma torácico surgió el gusto por el área en nuevas generaciones, de las cuales han egresado ocho cirujanos más hasta la fecha, todos ellos formados en el Centro Médico Nacional (CMN) 20 de Noviembre, del Instituto de Seguridad y Servicios Sociales de los Trabajadores del Estado (ISSSTE), después de concluir su entrenamiento en cirugía general, con periodos de 304 años. A continuación se proporciona un listado con sus nombres, el periodo en el cual se especializaron en cirugía cardiotorácica y el lugar donde actualmente laboran poniendo en alto los nombres de la Cruz Roja Mexicana y del 20 de Noviembre:

- Dr. Joaquín Rodolfo Zepeda Sanabria, 1990-1993 (CMN 20 de Noviembre y Hospital de Pediatría UMAE Siglo XXI).

- Dr. Francisco Rueda Ríos, 1992-1995 (Hospital del ISSSTE Tijuana).

- Dr. Hugo García Aranda, 1994-1997 (Hospital General de Zacatecas).

- Dra. Alma Angélica del Rocío Jiménez Godínez, 1995-1998 (Hospital Ángeles de Tijuana).

- Dra. María del Sol García Ortegón, 1998-2002 (CMN 20 de noviembre). 
- Dr. Jorge Villela Caleti, 1999-2003 (Hospital PEMEX Sur).

- Dr. Juan Carlos Vázquez Minero, 2001-2005 (INER y Cruz Roja DF).

- Dr. Manuel Fernando Rodríguez Ortega, 2003-2007 (Centro Médico ISSEMYM).

De estos subespecialistas, muchos migraron a sus lugares de origen, pero tres de ellos han trabajado en diferentes etapas en el Hospital Central de la Cruz Roja Mexicana, continuando la noble labor de los iniciadores del servicio de traumatología torácica: el Dr. Joaquín Rodolfo Zepeda Sanabria (1992-2006) en la guardia A, el Dr. Jorge Villela Caleti (2002-2004) en la guardia $A$, y el Dr. Juan Carlos Vázquez Minero (2005 a la fecha), responsable del servicio en el turno vespertino desde 2008.

Han transcurrido 62 años desde el inicio del proyecto del Dr. Pedro Alegría y su grupo, y se han tratado miles de pacientes con patología traumática torácica, buscando mejorar su atención, disminuir las complicaciones derivadas de sus lesiones y enseñar a los cirujanos generales el manejo adecuado de la patología traumática del tórax.

\section{El servicio de traumatología torácica en la actualidad}

El éxito de este servicio, en donde el cirujano cardiotorácico cumple con la función de cabeza, yace en la participación de todos los jefes de guardia y médicos de base de los diferentes turnos del servicio de cirugía general, junto con el residente de cirugía general, los cuales - durante las guardias - llevan a cabo los diagnósticos específicos, las intervenciones quirúrgicas y los manejos médicos especializados de urgencias de un sinfín de patologías traumáticas que involucran el tórax, tales como lesiones pulmonares, cardiacas, diafragmáticas, esofágicas, vasculares y de pared toráci$\mathrm{ca}$, efectuando procedimientos como toracotomías de urgencia y emergencia, y colocación de sondas endopleurales y su cuidado, minimizando las complicaciones más graves, como hemotórax retenidos y empiemas postraumáticos.

Los procedimientos mencionados han sido efectuados a lo largo de los 62 años de vida del servicio. Es imposible mencionar todos los nombres, pero es cierto que todos y cada uno de ellos han contribuido con su granito de arena para el fortalecimiento del servicio y la enseñanza del manejo oportuno del paciente con patología traumática del tórax. En la actualidad existe un especialista en cirugía cardiotorácica que, junto con los cirujanos generales y residentes, lleva a cabo la atención del paciente con trauma torácico.

\section{Conclusiones}

Si bien es cierto que no hay especialistas del área en todos los turnos, los cirujanos generales de la institución son capaces de resolver la patología aguda y urgente para dar paso al manejo posoperatorio y el seguimiento específicos de los pacientes por parte del encargado del servicio desde su fundación. De esta manera, la consulta se ha mantenido por 62 años ininterrumpidos, para dar seguimiento al paciente egresado del hospital.

En nuestro país hay muy pocos especialistas en cirugía torácica que se dediquen a la atención de pacientes traumatizados; no obstante, en el Hospital Central de la Cruz Roja Mexicana existe un servicio que cumple con esta misión fundamental desde hace 62 años. Se han atendido pacientes con lesiones pulmonares, cardiacas, traqueales, diafragmáticas, esofágicas y de grandes vasos; se han resuelto complicaciones como hemotórax coagulados, empiemas postraumáticos y fístulas broncopleurales, y se han derivado pacientes con estenosis traqueales, roturas bronquiales, disecciones de aorta y fallas cardiacas postraumáticas a centros especializados.

Esta labor no solo es de los especialistas en cirugía cardiotorácica, sino que también implica la participación de los cirujanos generales y residentes que trabajan hombro con hombro por el bienestar de los enfermos. A todos ellos, gracias.

\section{Bibliografía}

1. Carreón BR, Rodríguez PA. El inicio del manejo del traumatismo en la Ciudad de México. Gac Med Mex. 2005;141:161-3.

2. Rodríguez PA, Carreón BR. Cronología del manejo quirúrgico del trauma en México (900 A.C.-1917). Resumen histórico del trauma. Trauma. 2005;8:10-3.

3. Cruz Roja Mexicana. Una obra de mexicanos en beneficio de la humanidad. 85 años de la historia de la Cruz Roja Mexicana. México, D.F.: Cruz Roja Mexicana; 1995.

4. Cruz Roja Mexicana. 100 años gracias a ti. México, D.F.: Gustavo Casasola Ediciones; 2010.

5. Munguía $C D$, Ibarra PC, Rodríguez PM. Pioneros de la cirugía torácica mexicana. Gac Med Mex. 2011;147:342-9.

6. Alcántara HJ. Compendio de cronología quirúrgica mexicana. Cir Cir. 1951;19:1-21, 163-91.

7. Villagrán BJ. Apuntes sobre las heridas penetrantes de pecho complicadas de hematotórax, casos en los que está indicada la toracocentesis y ventajas de este tratamiento. Gac Med Mex. 1864;8:121-5.

8. Soriano MS. Estadística quirúrgica de las heridas penetrantes de tórax o vientre. Gac Med Mex. 1898;35:274-8.

9. Garza PA. Traumatología del tórax. México, D.F.: La Prensa Mexicana; 1962. p. VII-X.

10. Cruz Roja Mexicana. Libro de oro de la Cruz Roja. Edición conmemorativa. México, D.F.: Cruz Roja Mexicana; 1968.

11. Genis J. Testimonio. Trauma. 2006;9:5. 